\title{
A 33-35 kDa circulating antigen from Plasmodium falciparum
}

\author{
Neira de Dominguez ${ }^{1}$ and Alexis Rodriguez-Acosta ${ }^{2}$ \\ ${ }^{1}$ Laboratory of Biochemistry, Faculty of Pharmacy, Universidad Central de Venezuela, Ciudad Universitaria, Caracas, \\ Venezuela; \\ ${ }^{2}$ Section of Immunochemistry, Institute of Tropical Medicine, Universidad Central de Venezuela, Ciudad Universitaria, Caracas, \\ Venezuela
}

Key words: Plasmodium falciparum, soluble antigens, malaria, excretion antigens, hyperimmune serum, thermal stability

\begin{abstract}
Isolation and characterisation of Plasmodium falciparum (Welch, 1897) soluble antigens from infected patient plasma, Western blotting, thermal stability and ELISA assays using hyperimmune IgG-antimalaria antibodies was the main objective of this work. A circulating antigen of approximately Mr 33-35 kDa with good specificity and antigenicity, in the plasma of malarial patients was shown. Heating at $100^{\circ} \mathrm{C}$ did not destroy its antigenicity. When fractions highly enriched in the 33-35 kDa proteins were used in ELISAs, a seroreactivity in plasma obtained from primary-infected individuals was found. Controls from normal patients were always negative. The antigenic characteristics suggest that it may be included within the group of new described Plasmodium soluble antigens.
\end{abstract}

With the development of techniques for in vitro cultivation of Plasmodium falciparum (Welch, 1897) and the isolation of parasite soluble antigens from malaria patient's plasma, the opportunity presents itself to describe new Plasmodium antigens (Chougnet and Deloron 1992). The term "soluble antigen" includes a broad variety of antigens, a majority of which are probably released into the bloodstream of patients in vivo or in tissue culture supernatant in vitro upon schizont rupture and subsequent merozoite invasion.

Plasmodium falciparum excretion-secretion soluble antigens have been known since 1968 (McGregor et al. 1968). Several authors have studied their importance in protection against disease but they may be implicated in the development of immunopathological complications (Greenwood and Brueton 1974, Facer 1980, Urquihart 1992, Baltahazar-Guedes et al. 1995, Wahlgren et al. 1995). Recent advances in the identification and characterisation of antigens associated with different stages of the parasite suggest new opportunities for vaccination as a strategy for disease control. The potential of these substances as candidates for the development of diagnosis tools must also be indicated. Here we describe a novel antigen recognised by malarial serum. This $P$. falciparum soluble antigen was isolated from infected patient plasma and characterised by Western blotting, thermal stability and ELISA assays using hyperimmune IgG-antimalaria antibodies that could be associated with an active infection.

\section{MATERIALS AND METHODS}

Animals. New Zealand strain rabbits were used. All animals were well treated and care taken to avoid distress when blood samples were obtained. The investigation complied with the norms taken from the Guide for the Care and Use of Laboratory Animals, published by the US National Institute of Health (Anonymous 1985).

Sample collection. Blood samples from patients attending the Malaria Hospital at the Malariology Division of Bolívar City were collected. This is a city located in the northern part of Bolívar State, Venezuela. Many of the patients are migrant gold miners. They routinely spend between 20-30 days prospecting in the tropical forest where most of their infections (most assuredly) originate.

The region is a tropical area where the temperature and humidity allows transmission during most of the year. The main vector is Anopheles darlingi Root, essentially a nondomestic species that usually does not enter the houses, although when it does, it bites quickly. Malaria generally occurs with moderate incidence, rarely reaching the hyperendemic levels common in Africa.

A total of thirty patients with acute malaria (primary infection) were recruited for the antigen study. Sera from thirty patients who had suffered two or more malarial episodes were used as source of hyperimmune antibodies. For the investigation of the parasite antigens, a pool of plasma was used $(30 \mathrm{ml})$ belonging to the primary-infected patients included in this study. Parasitaemias of patients were always more than $2 \%$. Before being allowed to participate in the study, all the patients were required to give their oral informed consent.

Address for correspondence: A. Rodriguez-Acosta, Instituto de Medicina Tropical, Universidad Central de Venezuela, Apartado 47423, Caracas 1041A, Venezuela. Phone: ++58 2 6053558; Fax: ++58 2 6932094; E-mail: rodriguf@camelot.rect.ucv.ve 
Twenty $\mathrm{ml}$ blood samples were collected from each patient using $3.8 \%$ sodium citrate at a ratio of $1: 10 \mathrm{ml}$ and $100 \mu \mathrm{l}$ of a mixture of protease inhibitors (L-1-tosylamide 2-phenylethyl chloromethyl ketone [Sigma, Mo, USA] $0.15 \mathrm{mM}$, phenylmethylsulphonyl fluoride [Sigma] $200 \mathrm{mM}$, ethylenediaminetetraacetic acid $0.2 \mathrm{mM}$ in PBS). Plasma $(30 \mathrm{ml})$ from thirty primary-infected patients were pooled and used to investigate parasitic antigens. The Ig-free plasma (immunoglobulins were previously ammonium sulphate precipitated) was dialysed against PBS pH 7.4 and concentrated to a volume of $5 \mathrm{ml}$ inside a dialysis bag using Aquacide II (Calbiochem, USA). The entire procedure was performed at $4{ }^{\circ} \mathrm{C}$.

Anti-whole-parasite serum. The infected erythrocytes (20 $\mathrm{ml}$ ) derived from cultures with at least 3\% parasitaemia were suspended in PBS, $\mathrm{pH} 7.2$, and were centrifuged at $300 \mathrm{~g}$ for 10 minutes at $4^{\circ} \mathrm{C}$. After being washed with PBS three times, the erythrocytes were lysed with saponin (Sigma, Mo, USA) in a final concentration of $0.1 \%$ for 15 minutes at room temperature, washed and resuspended again in $0.5 \mathrm{ml}$ of PBS containing $0.1 \%$ of the mixture of protease inhibitors (crude preparation of parasite extracts). This antigen was used to immunise the rabbits and obtain anti-whole-parasite serum, as described (Hudson and Hay 1980).

Immune sera. Human anti- $P$. falciparum immune sera from ten Yanumana Amerindians living in a malaria-hyperendemic area (Amazonas State, Venezuela), and sera $(30 \mathrm{ml})$ from thirty patients who had suffered two or more malarial episodes were pooled and used to isolate parasite antigens. These mixtures were treated with the appropriate volume of saturated ammonium sulphate solution to precipitate immunoglobulins. Corresponding control sera from uninfected individuals at non-malaria-endemic area were obtained. Control eluate attained by affinity chromatography in the SDS gel and the ELISA analysis were tested.

Malarial antigen immunoaffinity separation. Immunoglobulin-free plasma (400 $\mathrm{mg}$ protein) from patients with acute $P$. falciparum infection as a source of antigens was loaded on Sepharose-4B-hyperimmune human-IgG-anti- $P$. falciparum columns. The columns with PBS were washed until $\mathrm{OD}_{280}=0$. The fraction bound to the column was eluted with $0.1 \mathrm{M}$ glycine- $\mathrm{HCl}$ buffer, $\mathrm{pH} 2.6$ and concentrated (Fig. $1)$.

Parasite culture. Isolates from $P$. falciparum infected patients (Bolívar State, Venezuela) were procured. Parasites were cultured in non-synchronised cultures of all stages with human erythrocytes ( 0 group) at $2 \%$ haematocrit in RPMI 1640 medium and 10\% human serum (Trager and Jensen 1976). The medium was changed every 24 hours, then treated with a cocktail of protease-inhibitors as previously mentioned, centrifuged to remove red blood cells (RBC) and debris and stored at $-70^{\circ} \mathrm{C}$ until used.

Preparation of IgG anti-culture supernatant. A volume of approximately $300 \mathrm{ml}$ of culture supernatant was concentrated 30 times by dehydration inside a dialysis bag with Aquacide II. The concentrate was $33 \%$ ammonium sulphate precipitated, to eliminate the human serum immunoglobulins present in the culture medium. The Ig-free supernatant was dialysed against $0.05 \mathrm{M}$ Tris- $\mathrm{HCl}$ buffer, $\mathrm{pH}$ 7.6 for 16 hours at $4{ }^{\circ} \mathrm{C}$, concentrated and used to prepare anticulture supernatant serum by inoculating rabbits as described
(Hudson and Hay 1980). The sera from immunised rabbits were used to prepare IgG by $33 \%$ ammonium sulphate precipitation and DEAE cellulose (Sigma, Mo, USA) ionic exchange chromatography (Hudson and Hay 1980). This IgG $(100 \mathrm{mg}$ protein $/ \mathrm{ml})$ was absorbed on a mixture of red blood cells (RBCM) type $0^{+}, \mathrm{A}^{+}$and $\mathrm{B}^{+}(1: 1: 1)(1 \mathrm{ml} \mathrm{RBCM}: 3 \mathrm{ml}$ $\mathrm{IgG})$ for 2 hours at $4^{\circ} \mathrm{C}$, and centrifuged at $200 \mathrm{~g}$ for 10 minutes. This supernatant was exhaustively absorbed on a column of Sepharose-4B-human serum and the eluate concentrated using Aquacide II. Purified immunoglobulins were used to perform the ELISA analysis of the antigens.

Electrophoresis. Electrophoresis was carried out using a Dual Mini Slam Kit AE-6450 (Atto Corporation, Tokio, Japan) chamber (Laemmli 1970).

Gels at $7.5 \%$ with stacking gel at $4.5 \%$ were prepared. The samples (antigenic fraction) to be analysed were dissolved in a proportion of $1: 1$ in the solubiliser solution: $0.5 \mathrm{M}$ Tris- $\mathrm{HCl}$, $\mathrm{pH} 6.8$, with $10 \%$ (w/v) SDS, $10 \%$ (v/v) B-mercaptoethanol, $10 \%(\mathrm{v} / \mathrm{v})$ glycerol and $0.05 \%(\mathrm{w} / \mathrm{v})$ bromophenol blue, and heated at $100^{\circ} \mathrm{C}$ for 10 minutes. Gels were stained with Coomassie blue.

Western blotting. Immunoblotting technique (Towbin et al. 1979) was used. The electrophorised proteins were blotted onto nitrocellulose filter using a semi-dry electrotransfer chamber with graphite electrodes (Atto Corporation, Tokio, Japan). The filters were analysed by immunostaining, using serum containing specific antibodies (human IgG-anti- $P$. falciparum). Once the transfer had been completed, the nitrocellulose sheets were blocked with $0.03 \%$ solution of Tween 20 in PBS for one hour. After washing with $0.01 \%$ Tween 20 in PBS $(4 \times 15$ minutes $)$, the strips were incubated for one hour on a rocker at room temperature with the antibodies diluted in 5\% foetal calf serum (FCS) in PBS, as indicated earlier. After washing $(4 \times 15$ minutes $)$, the strips were incubated with the secondary antibody (anti-human-IgGperoxidase) appropriately diluted in PBS for two hours at room temperature, then washed and developed with diaminobenzidine (DAB, Sigma, USA) for 1 or 2 minutes until the immunoreactive proteins were stained clearly. To stop the reaction, distilled water was used.

Thermal stability of the antigen 33-35 kDa separated by affinity chromatography. The fraction Sepharose-4Bhyperimmune-IgG-malaria linked was incubated at temperatures of 60,80 and $100^{\circ} \mathrm{C}$ for periods of $2,5,10$ and 20 minutes (Wilson et al. 1969).

Sample percentage residual antigenicity (RA) was evaluated by ELISA assay (Engvall and Perlmann 1971).

The \% RA was calculated using the relationship:

$$
\% \mathrm{RA}=\frac{\mathrm{A}_{1}}{\mathrm{~A}_{2}} \times 100
$$

$\% \mathrm{RA}=$ residual antigenicity.

$\mathrm{A}_{1}=$ difference of $\mathrm{OD}_{450}$ between positive and negative sera, after treatment.

$\mathrm{A}_{2}=$ difference of $\mathrm{OD}_{450}$ between positive and negative sera, before treatment. 
Enzyme-linked immunosorbent assay (ELISA). We followed the methodology of Engvall and Perlmann (1971) and Hudson and Hay (1980), as modified for microassay (Voller et al. 1978), using a direct (Assay 1), and an indirect (Assay 2) (immunoadsorption of the antigen) assay. Different concentrations of $P$. falciparum antigen fractions obtained by affinity chromatography purification were used. The direct tests were carried out with the antigen $(6 \mu \mathrm{g} / \mathrm{ml})$ in 96 flatbottom-well microtiter plates placed overnight at $4^{\circ} \mathrm{C}$. Plasma from patients with acute ( $>2 \%$ parasitaemia) $P$. falciparum malaria were obtained. The conjugated anti-human-IgGperoxidase as a secondary antibody was used. 3,3'-diaminobenzidine peroxidase (Sigma, Mo, USA) was used as substrate. The reaction was stopped by adding $4 \mathrm{~N} \mathrm{H}_{2} \mathrm{SO}_{4}$ after 30 minutes and the absorbance was measured at $450 \mathrm{~nm}$. All samples were with both positive and negative controls.

Indirect tests in 96 flat-bottom well microtiter plates were incubated for 2 hours at $37^{\circ} \mathrm{C}$ with the rabbit polyclonal antiwhole $P$. falciparum antibodies. After washing three times with a solution of $0.05 \%$ PBS-Tween, plates were incubated overnight at $4^{\circ} \mathrm{C}$ with $P$. falciparum antigens at $6 \mu \mathrm{g} / \mathrm{ml}$ in carbonate buffer $\mathrm{pH}$ 9.6. The rest of the process was performed as for the direct test.

Protein determination. The protein determination method followed that of Lowry et al. (1951).

Statistical methods. Statistical comparisons were made utilising unpaired Student's t-test $(\mathrm{P}=0.05)$.

\section{RESULTS}

The antigenic fraction separation by affinity chromatography with Ig-antimalaria ligands from hyperimmune patients is shown in Fig. 1.

Fig. 2 shows the polyacrylamide gel electrophoresis analysis of antigens retained by a Sepharose-4B-IgGantimalaria column and indicates the two bands of $\mathrm{Mr}$ 33-35 kDa seen in a pool of samples of $P$. falciparum primary-infected individuals.

Western blot analysis of the antigens retained by a Sepharose-4B-IgG-antimalaria column is shown in Fig. 3. Peroxidase-conjugated-IgG anti-P. falciparum immunological analysis shows the two bands of Mr 33-35 $\mathrm{kDa}$ seen in a pool of samples of $P$. falciparum primaryinfected individuals analysed.

To corroborate the presence of antibodies against the 33-35 $\mathrm{kDa}$ proteins in sera from primary-infected patients, two types of immunoassays (ELISA) were conducted:

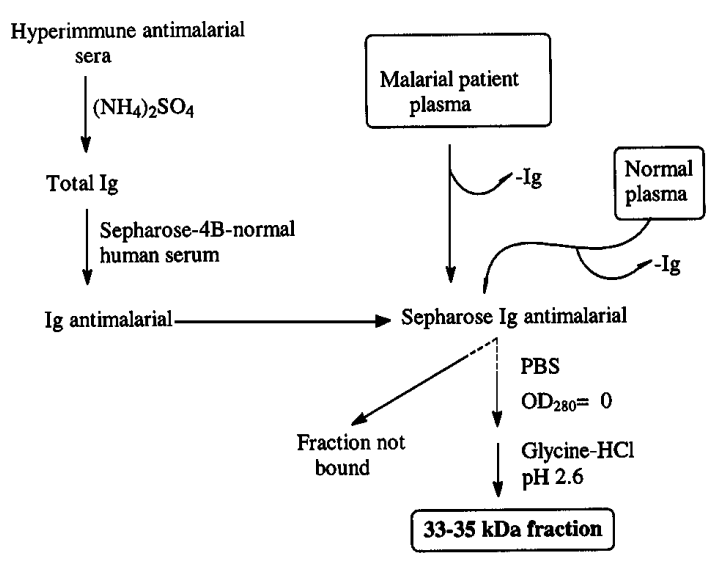

Fig. 1. Procedure for separation of antigenic fractions from malarial patient plasma by affinity chromatography.

In the direct assay (Fig. 4), IgG antibodies were evaluated against the antigens present in the pooled plasma of the group of patients chosen; $\mathrm{OD}=0.139$ as a positive limit $(X \pm$ S.D. $)$ was established. The mean value obtained from the group of patients (primaryinfected patients) was $0.351 \pm 0.075$, and it was significantly higher than for healthy patients (control sera) $(P \leq 0.01)$. The sensitivity for acute sera (primaryinfected) from patients was $100 \%$ (Table 1 ).

In the indirect assay (Fig. 5) a value of 0.172 units OD was established as positive limit ( $\bar{X} \pm$ S.D. $)$. As in Assay 1, differences with respect to mean values of the experimental group between infected and non-infected patients (control sera) were significantly different $(\mathrm{P} \leq$ $0.01)$. The serum sensitivity for the group of primaryinfected patients was also $100 \%$ (Table 1). Control eluate obtained by affinity chromatography tested in the SDS gel and the ELISA analysis were always negative.

Regarding thermal stability of the antigens, the percentage of residual antigenicity (\% RA) values are shown in Table 2. These results indicate that the antigenicity of the preparation is maintained higher than the $80 \%$ of the control activity, even in the case of the 33$35 \mathrm{kDa}$ fraction treatment at $100^{\circ} \mathrm{C}$ for 5 minutes. These results indicate the thermal stability of the antigens under analysis.

Table 1. 33-35 kDa antigen mean values ( $\bar{X} \pm$ S.D.) and sensitivity (\%) obtained by two ELISA methods.

\begin{tabular}{|l|c|l|c|l|}
\hline & \multicolumn{2}{|c|}{ Direct assay } & \multicolumn{2}{c|}{ Indirect assay } \\
\hline Controls & \multicolumn{2}{|c|}{ C: 0.139} & \multicolumn{2}{c|}{ C: 0.172} \\
\hline $\begin{array}{l}\text { Primary-infected } \\
\text { patients }\end{array}$ & $\begin{array}{c}\bar{X}: 0.351 \pm 0.075 \\
\mathrm{n}=16\end{array}$ & S: $100 \%$ & $\begin{array}{c}\bar{X}: 0.417 \pm 0.076 \\
\mathrm{n}=16\end{array}$ & S: $100 \%$ \\
\hline
\end{tabular}

$\mathrm{C}-$ cut off $(X+3$ S.D. $) ; \mathrm{S}-$ sensitivity; $\mathrm{n}$ - number of patients 


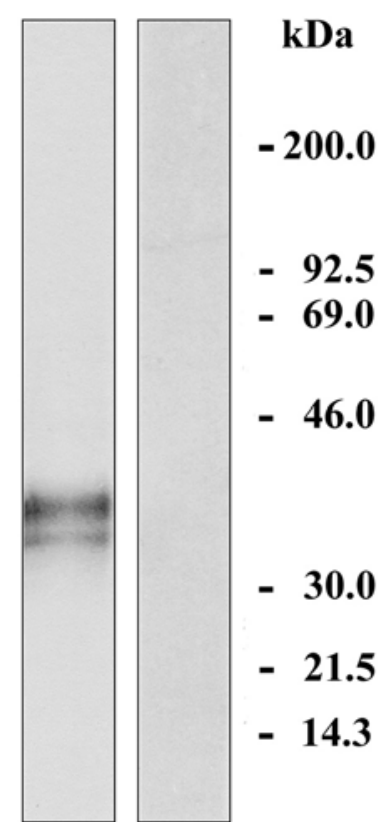

Fig. 2. Polyacrylamide gel electrophoresis of the antigenic fraction (AF) obtained by affinity chromatography from Plasmodium falciparum primary-infected patient plasma. The $\mathrm{AF}$ electrophoretic separation was performed in polyacrylamide-SDS gels at 7.5\% under reduced conditions: $\mathbf{a}-33-35$ $\mathrm{kDa}$ antigenic fraction; $\mathbf{b}$ - normal plasma.

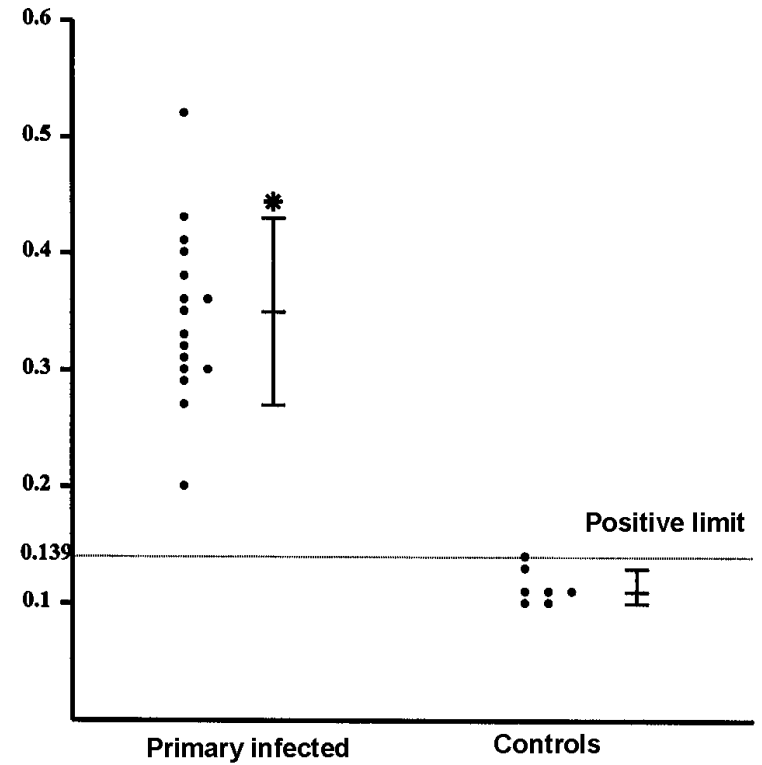

Fig. 4. Determination of $\operatorname{IgG}$ anti-antigenic fraction in the plasma of malaria patients by direct ELISA. Left: primaryinfected patients, $\mathrm{n}=16$. Right: controls, $\mathrm{n}=7 .{ }^{*} \mathrm{P} \leq 0.01$.

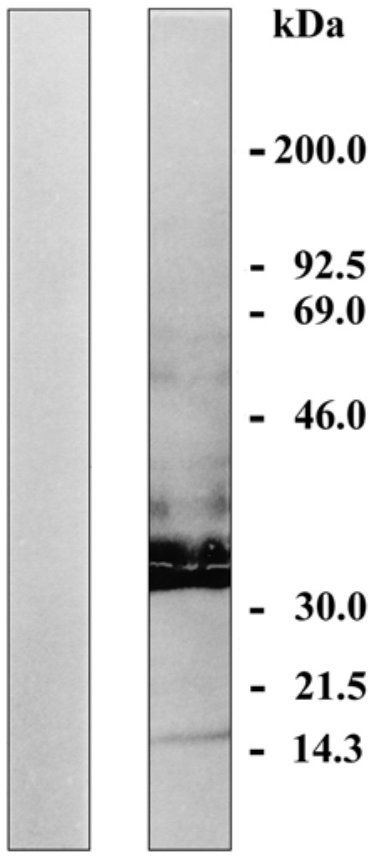

Fig. 3. Western blot analysis of the antigenic fraction (AF) present in Plasmodium falciparum primary-infected patient plasma linked to Sepharose-4B-IgG (hyperimmune selected) antimalaria. The AF electrophoretic separation was carried out in polyacrylamide-SDS gels at $7.5 \%$ under reduced conditions. The material transferred to nitrocellulose was revealed by incubation with: a - normal human serum/antihuman-IgG-peroxidase; b - hyperimmune serum/anti-humanIgG-peroxidase.

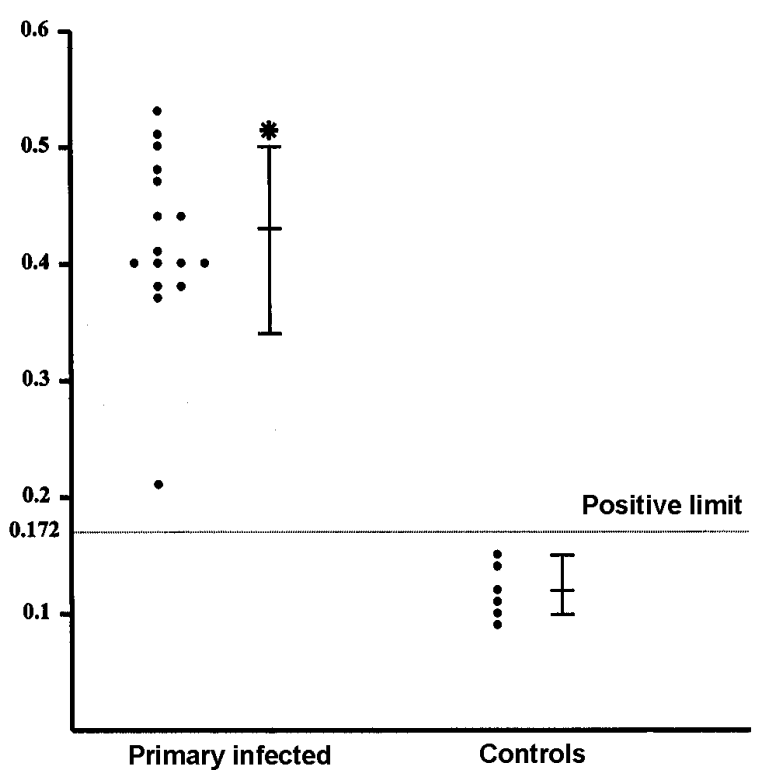

Fig. 5. Determination of $\operatorname{IgG}$ anti-antigenic fraction in the plasma of malaria patients by indirect ELISA. Left: primaryinfected patients, $\mathrm{n}=16$. Right: controls, $\mathrm{n}=6$. ${ }^{*} \mathrm{P} \leq 0.01$. 


\section{DISCUSSION}

The antigen captured by the rabbit polyclonal anti- $P$. falciparum antibodies in the ELISA indirect tests provided important evidence about the parasitic nature of the antigen. The inclusion of anti-whole-parasite polyclonal antibodies has proved to be a valuable tool in the characterisation of this parasite antigen.

Other authors (Wilson and Ling 1979, Anders et al. 1983) have also found the main characteristics of exoantigens such as solubility, thermostability and molecular weight. In the present study some of these characteristics were verified for the 33-35 kDa exoantigen of $P$. falciparum. Molecular weight comparisons among exoantigens are very difficult to procure because they can vary from 120 to $250 \mathrm{kDa}$ (WHO 1984). Jepsen (1983) reported lower molecular weight ranging from $97 \mathrm{kDa}$ to $37 \mathrm{kDa}$. In our work, a molecular weight of 33-35 $\mathrm{kDa}$ was found by SDSPAGE. ELISA analysis searching for antigens in patient plasma showed the presence of 33-35 kDa molecules.

As was described in the Western blot results, the fraction retained by Sepharose-4B-antimalaria indicated the two bands of $\mathrm{Mr} 33-35 \mathrm{kDa}$ in the plasma of $P$. falciparum primary-infected patients and recognised by hyperimmune anti- $P$. falciparum serum.

The thermostability study showed that this antigen is probably related to S antigens (Wilson and Ling 1979). The results obtained by direct and indirect ELISA indicate the presence of $\operatorname{IgG}$ antibodies against the 33$35 \mathrm{kDa}$ proteins. The adequate correlation obtained with SDS-PAGE and ELISA indicates that 33-35 kDa antigens are closely related to total precipitating exoantigens. Control eluate obtained by affinity chromatography tested in the SDS gel and the ELISA analysis were always negative, confirming the $33-35 \mathrm{kDa}$ protein as a malarial antigen. ELISA is a technique that can be used to follow the dynamics of this antigen release into the patient plasma without the requirement to partially purify each sample. Several authors have demonstrated that antigens are released during the later stages of the parasitic cell cycle (Wilson et al. 1969, Wilson and Bartholomew 1975). We found that 33-35 $\mathrm{kDa}$ proteins are released during 24 hours period that corresponds to free merozoites and erythrocyte reinvasion.

Identification and characterisation of new malaria antigens should contribute to the improvement of malaria diagnosis in individuals and population groups, in anti-malaria vaccine evaluation and in the measurement of the impact of malaria control campaigns. Affinity chromatography using Ig-anti-malaria ligands of hyperimmune patients enabled the separation of the antigenic fractions retained by these specific antibodies, from plasma of the malarial patients selected for this study. The immune-adsorbents were pre-run by
Table 2. Percentages of residual antigenicity of heat-treated antigenic fraction, calculated as OD values obtained by ELISA $(\bar{X} \pm$ S.D. of 6 determinations; antigenicity of untreated fraction $=100 \%)$.

\begin{tabular}{|c|c|c|c|}
\hline & \multicolumn{3}{|c|}{ Residual antigenicity (\%) } \\
Temperature $\left({ }^{\circ} \mathrm{C}\right)$ \\
\hline 0 & 60 & 80 & 100 \\
\hline 2 & $100 \pm 6.0$ & $100 \pm 6.7$ & $100 \pm 6.6$ \\
5 & $96 \pm 5.8$ & $89 \pm 5.8$ & $89 \pm 5.0$ \\
10 & $98 \pm 5.0$ & $83 \pm 6.4$ & $81 \pm 3.5$ \\
20 & $88 \pm 7.0$ & $90 \pm 4.0$ & \\
\hline
\end{tabular}

affinity in a column of Sepharose-normal human serum to eliminate those autoantibodies directed against elements of the normal human tissues in the malaria patient plasma (Ritter 1993). Studies of P. falciparum cultures indicate that the antigens excreted by the intraerythrocytic schizont stage are immunochemically similar to those detected in host infected serum (Wilson and Bartholomew 1975, Ramos et al. 1989), but there are very few reports concerning their characterisation. Based on thermal stability, P. falciparum $33-35 \mathrm{kDa}$ proteins may be classified within the group of the Type $\mathrm{S}$ excretion antigens. The only antigen with a relative mass of $33 \mathrm{kDa}$ that has been found was described by Ramos et al. (1989). However, this antigen is different from the 33-35 $\mathrm{kDa}$ proteins since its antigenicity was damaged by treatment at $65^{\circ} \mathrm{C}$, and it was classified as a Type La soluble antigen (Ramos et al. 1989). Its similarity to known Type $\mathrm{S}$ antigen and the presence of two bands (33-35 $\mathrm{kDa})$ suggest that the antigen described here is definitely a different molecule to that of similar molecular weight characterised previously. If this antigen were to be used in a diagnostic assay, it would have the advantage of thermoresistance, so making it attractive to commercial development in a kit for use in tropical countries under extreme conditions of temperature.

In conclusion, the reactivity of 33-35 $\mathrm{kDa}$ proteins in ELISA assay suggests that this is a parasite-excreted antigen as it is not found in normal serum or in the serum of malaria patients without active infection. The characteristics of this soluble antigen, with good antigenicity and thermoresistance, indicate it could be useful as antigenic marker of active $P$. falciparum infections.

Acknowledgements. This work received financial support from the Consejo de Desarrollo Científico y Humanístico and Instituto de Investigaciones Farmaceúticas de la Universidad Central de Venezuela. We thank Dr. Stephen Tillet and an anonymous referee for reviewing the English of the manuscript. 


\section{REFERENCES}

ANDERS R.F., BROWN G.V., EDWARDS A. 1983: Characterization of an S-antigen synthesised by several isolates of Plasmodium falciparum. Proc. Natl. Acad. Sci. U.S.A. 80: 6652-6656.

ANONYMOUS 1985: Principles of Laboratory Animal Care. National Institute of Health, Maryland, USA, $96 \mathrm{pp}$.

BALTAHAZAR-GUEDES H.C.F., FERREIRA D.A., CRUZ M.F., MONTENEGRO J., DANIEL-RIBEIRO C.T. 1995: Malaria diagnosis: identification of an anti-40-kDa polypeptide antibody response associated with active or recent infection and study of the $\mathrm{IgG} / \mathrm{IgM}$ ratio of antibodies to blood-stage Plasmodium falciparum antigens. Parasitol. Res. 81: 305-309.

CHOUGNET C., DELORON P. 1992: Is immunity to malaria really short-lived? Parasitol. Today $8:$ 375-376.

ENGVALL E., PERLMANN P. 1971: Enzyme-linked immunosorbent assay (ELISA) quantitative assay for immunoglobulin G. Immunochemistry 8: 871-876.

FACER C. 1980: Direct Coombs antiglobulin reaction in Gambian children with Plasmodium falciparum malaria. II. Specificity of erythrocyte bound IgG. Clin. Exp. Immunol. 39: 279-288.

GREENWOOD B., BRUETON N. 1974: Complement activation in children with acute malaria. Clin. Exp. Immunol. 18: $267-272$

HUDSON L., HAY F.C. 1980: Practical Immunology. Blackwell Scientific Publications, Oxford, $126 \mathrm{pp}$.

JEPSEN S. 1983: Inhibition of in vitro growth of Plasmodium falciparum by purified antimalarial human $\operatorname{IgG}$ antibodies. Isolation of target antigens from culture supernatants. Scand. J. Immunol. 18: 8631-8636.

LAEMMLI U.K. 1970: Cleavage of structural proteins during the assembly of the head of bacteriophage T4. Nature (London) 227: 680-685.

LOWRY O.H., ROSEMBROUGH N.J., FARR A.L., RANDALL R.J. 1951: Protein measurement with the folin phenol reagent. J. Biol. Chem. 193: 265-272.

McGREGOR I., TURNER M., WILLIAMS K., HALL P. 1968: Soluble antigens in the blood of African patients

Received 24 January 2000 with severe Plasmodium falciparum malaria. Lancet 1 (7548): 881-884.

RAMOS M.I., OMATA Y., IGARASHI M., NAKABAYASHI T. 1989: Plasmodium falciparum: characterization of a 33-kDa soluble antigen. Exp. Parasitol 68: 297-307.

RITTER K. 1993: Prolonged haemolytic anaemia in malaria and autoantibodies against triosephosphate isomerase. Lancet 342: 1333-1334.

TOWBIN H., STAEHLIN T., GORDON J. 1979: Electrophoretic transfer of proteins from acrylamide gels to nitrocellulose sheets: procedure and some applications. Proc. Natl. Acad. Sci. U.S.A. 76: 4350-4354.

TRAGER W., JENSEN J.B. 1976: Human malaria parasites in continuous culture. Science 193: 673-676.

URQUIHART A.D. 1992: Phagocytic cells in human cerebral malaria. J. Infect. Dis. 166: 937-942.

VOLLER A., BIDWELL B., HOLDT G., ENGVALL E. 1978: A microplate method of enzyme linked immunosorbent assay and its applications to malaria. Bull. WHO 51: 209-211.

WAHLGREN J., ABRAMS J.S., FERNANDEZ V., BEJARANO M.T., AZUMA M., TORII M., AIKAWA M., HOWARD R.J. 1995: Adhesion of Plasmodium falciparum-infected erythrocytes to human cells and secretion of cytokines (IL-1- $\beta$, IL-1RA, IL-6, IL-8, IL-10, TGF $\beta, T N F \alpha$, G-CSF, GM-CSF). Scand. J. Immunol. 42: 626-636.

WHO 1984: Recent progress in the development of malaria vaccines: memorandum from a WHO meeting. TDR/ IMMAL/SWG (6) 84.3. Geneva, Switzerland.

WILSON R.J.M., BARTHOLOMEW R.K. 1975: The release of antigens by P. falciparum. Parasitology 71: 183-192.

WILSON R.J.M., LING I.T. 1979: Fractionation and characterization of Plasmodium falciparum antigens. Bull. WHO 57: 123-126.

WILSON R.J.M., McGREGOR I.A., HALL P., WILLIAMS K., BARTHOLOMEW R. 1969: Antigens associated with Plasmodium falciparum infections in man. Lancet 2 (7613): 201-205.

Accepted 25 May 2000 